\title{
Management of qualitative change in small and medium-sized enterprises: a kaleidoscope of theoretical and empirical research
}

\author{
Matthias Fink • Hermann Frank • \\ Katherine Gundolf • Norbert Kailer
}

Published online: 23 December 2014

(C) Springer-Verlag Berlin Heidelberg 2014

Change has always fascinated scholars across disciplines and numerous theories and models of organizational change and development have been published (Weick 1979; Van de Ven and Poole 1995). In management science, change has long been conceptualized as the opposite of stability (Adler et al. 2009; March 1991; Nelson and Winter 1982; March and Simon 1958). Only recently management theory conceptually overcame this dualism and established an understanding of duality of change and stability. In this thinking it is the interplay of change and stability that fosters critical developments in firms such as innovation, internationalization and strategic renewal (Moshe 2010).

Understanding stability and change as the two sides of the same coin opens the door for a more contextualized investigation of change in firms (Cohen 2007). This allows for research into the complex and sometimes paradoxical relation between

\footnotetext{
M. Fink $(\bowtie)$

Institute for Innovation Management, JKU Johannes Kepler University Linz, Altenbergerstraße 69, 4040 Linz, Austria

e-mail: matthias.fink@jku.at

M. Fink

Institute for International Management Practice, ARU Cambridge, East Road, Cambridge CB1 1PT, UK

H. Frank

Institute for SME Management and Entrepreneurship, WU Vienna University of Economics and Business, Welthandelsplatz 1, 1020 Vienna, Austria

K. Gundolf

Montpellier Business School, 2300, avenue des Moulins, 34185 Montpellier Codex 4, France

N. Kailer

Institute for Entrepreneurship and Organizational Development, JKU Johannes Kepler University Linz, Altenbergerstraße 69, 4040 Linz, Austria
} 
stability and change (Sutherland and Smith 2011; Van Gestel and Hillebrand 2011). The extent of stability and change in each development process is contingent on the content of change, the organizational setting and the external framework conditions (Mejia-Morelos et al. 2013).

Change comprises a qualitative and a quantitative dimension. Especially the interplay between quantitative and qualitative change seems to provide potential for management research. The basic idea might be illustrated by a popular saying: "Just because everything is different doesn't mean anything has changed." Firms might grow, but they just do more of the same. In contrast, they might not change in size or quantity of their activity, but substantially change in the way they think or do their business.

Even though quantitative and qualitative aspects of change can be distinguished analytically, in explaining change comprehensively both dimensions need to be taken into consideration (Davidsson et al. 2010). Both aspects of change necessarily require a point of reference. But there is no absolute stability over time, because the same situation will never occur twice. Nevertheless, the fiction of stability as a joint point of reference makes it possible to identify the magnitude and nature of change. As soon as research tries to find explanations for quantitative change, the explanations necessarily draw on the nature of change. Thus it is the qualitative aspects of change that need to take centre stage in research on change in organizations. Explanations for the growth of a small and medium-sized enterprise (SME) from 10 to 60 employees will not be feasible without drawing on the related qualitative aspects.

SMEs are especially well suited to investigate qualitative change. Due to the greater amplitude of change relative to their size, quantitative change leads to more prominent qualitative change. As most firms start as SMEs, qualitative change more likely happens for the first time in this type of firm. Thus, SMEs face distinct challenges in managing qualitative change. More than in larger firms, which may have established routinized practices for managing qualitative change, smaller firms tend to struggle with handling new management demands along their developmental path. At the same time, SMEs often lack the capacity needed to reflect on their change experiences and thus do not tap the full potential of learning from these experiences. SMEs that cannot revert to change routines might easily be threatened in their existence. Interestingly, even if these distinct challenges for SMEs and their practical relevance are unquestionable, research on the qualitative change in SMEs is still limited.

In this special issue we aim at contributing to reduce this gap. For that, from different fields of management science, we draw together firm-level research with a focus on established SMEs going through phases of qualitative change. While structural, strategic and/or cultural changes may result in or be caused by quantitative change, a merely quantitative change is not within the scope of this special issue.

The vivid response to our call allowed us to employ a rigid selection process that resulted in an exciting portfolio of studies that follow most different theoretical and methodological approaches and that span different fields of management science. The selection comprises empirical studies employing a quantitative and qualitative 
methodical approach, as well as research-driven literature reviews and work involving the development of new theoretical frameworks. As we believe that studies that challenge established wisdom are most beneficial for scientific progress, the selected contributions take a critical rather than a conformist stance and problematise assumptions of well-acknowledged management research. In sum, the collection of studies presented in this special issue provides a much needed contribution to the current discourse and the body of knowledge on the management of qualitative changes in SMEs.

The first two contributions set the stage in addressing the two key elements of this special issue: SME and qualitative change. In their paper titled "Problematization and Conceptualization of 'Entrepreneurial SME Management' as a Field of Research: Overcoming the Size-based Approach" Hermann Frank and Dietmar Rößl challenge the established understanding of what an SME is and thus question the common wisdoms about the characteristics of SME management. They apply a prescriptive approach which delineates SME management as a field of research that investigates decisions and processes in companies regardless of their size and age and are of interest as long as they have not become routine yet. Thus, the authors' main contribution is the idea to put qualitative change at the heart of their approach to SME management.

The second contribution by Zulaicha Parastuty, Erich Schwarz, Robert Breitenecker and Rainer Harms titled "Organizational Change: A Review of Theoretical Conceptions that Explain how and why Young Firms Change" provides a concise review and classification of theories on organizational change with a focus on early phases of firm development. Especially the main assumptions of the theories and the way they conceptualise the nature and reason of change are analysed, which leads to a discussion of the boundary conditions of their applicability. Thus, the authors add to the understanding if the established theories of organizational development can serve, also in SMEs, as a basis for research on qualitative change management.

The remaining contributions address specific aspects of qualitative change in SMEs including family businesses. The next two papers especially contribute to an empirically informed theory development in the realm of SME management. Wolfgang Güttel, Stefan Konlechner and Julia Trede, in an article titled "Standardized Individuality vs. Individualized Standardization: The Role of the Context in Structurally Ambidextrous Organizations" show how managing the interplay of organizational structure and context enables a globally operating SME to combine exploration and exploitation. They draw on an empirical case-study to show that the establishment of a common frame of reference makes it possible for organizational members to integrate practices of exploration and exploitation. The authors' main contribution to theory is the introduction of the idea of a "secondorder"-balance between exploration and exploitation that allows to productively use the tension between incremental/radical innovation and adaptability/alignment.

The paper "Working on a Dream: Sustainable Organizational Change in SMEs" by Isabella Hatak, Alexander Zauner and Arne Floh employs a Delphi study in medium-sized Austrian wineries to understand how qualitative change associated with a shift to sustainability unfolds on the firm level. The authors develop a multilayer process model that differentiates between unfreezing, changing and refreezing 
processes, which can provide orientation for managing and researching qualitative change in SMEs. Their findings highlight the critical role of the interplay between change and stability across the different layers throughout the development process. Especially the empirically based model of change may inform future research and contributes to a more sophisticated understanding of the process of qualitative change in SMEs.

Further contributions address specific and highly relevant aspects of managing qualitative change in SMEs. Specifically, the next two papers investigate how control impacts on change in SMEs. "Ready for a Crisis? How Supervisory Boards Affect the Formalized Crisis Procedures of Small and Medium-sized Family Firms in Germany" is a paper by Pedram Faghfouri, Nils Kraiczy, Andreas Hack and Franz Kellermanns that analyses the relation between family ownership and formalized crisis procedures in 150 SMEs. The paper highlights the need to establish formal crisis procedures because not being prepared for crisis may threaten the survival of family firms. The authors address a key but often neglected topic and significantly contribute to ongoing discourse on effective structures for family firms.

In their contribution titled "The Auditor as a Change Agent for SMEs: The Role of Confidence, Trust and Identification" Ewald Aschauer and Andrea Moro turn their attention to the auditor as an important external business partner for an SME in mastering qualitative change. Based on 166 German auditor-SME client dyads they find an auditor's identification with the client firm and the auditor's confidence in the client management to be highly relevant and auditor's trust in the client as not relevant to the level of qualitative change in the client firm. The key contribution of this study is the insight that external control procedures based on quantitative indicators only induce qualitative change in the firm as long as the social relationship between the controlling and the controlled is supportive.

The next paper also addresses a relation with external stakeholders. Ricarda Bouncken, Robin Pesch and Sascha Kraus in their study "SME Innovativeness in Buyer-Seller Alliances: Effects of Entry Timing Strategies and Inter-organizational Learning" focus on SMEs' innovation timing and the associated market entry strategy to initiate change. They investigate whether to take a pioneer or a follower strategy for the innovation's market entry based on a longitudinal sample of 169 established SMEs. They find that SMEs profit from a pioneer strategy and may even compromise their performance with a follower strategy. The results add to our understanding of how timing matters for qualitative change in SMEs to positively impact on firm performance.

Tim Gittins, Richard Lang and Magdolna Sass contribute their study titled "The Effect of Return Migration Driven Social Capital on SME Internationalisation: A Comparative Case Study of IT Sector Entrepreneurs in Central and Eastern Europe". They argue that political and economic transition has facilitated outward migration, which — with some time lag-results in return migrants who acquire social capital abroad. The return migrants influence qualitative change in the business practice in the form of entrepreneurship, organizational human capital and SME internationalisation. The study contributes to our understanding of qualitative change by revealing that return migrants' social capital is crucial for inducing the internationalisation of the firms in their country of origin. 
Finally, the study "Navigating a Family Business through a Changing Environment-A Longitudinal Study" by Sabine Reisinger and Johannes Lehner draws on extensive data from a longitudinal case study of a family firm. They provide insights into how an entrepreneurial couple integrate their complementary skills and mindsets in order to balance the tension between stabilisation and innovation. They identify distinct dynamic capabilities for family firms managed by a couple whose interpersonal trust allows for bridging the gap between conflicting demands in dynamic environments.

In a nutshell, qualitative change management is a core element of the academic field of SME management. The theories in this field do not have to be developed from scratch. At the same time, they cannot be simply adopted from general research on organizational change, but have to be assessed for their applicability in the SME context. Two possible avenues for identifying and adopting theories relevant for research on qualitative change in SMEs are the concept of "secondorder"-balance between exploration and exploitation and the multi-layer concept of parallel micro-processes of change. Regarding specific aspects of qualitative change, relations to external stakeholders and SME owner-managers have been identified to trigger and feed change. Further, internal and external control has been shown to play a critical role for translating change into firm performance.

We are convinced that this collection of studies will positively contribute to the on-going discourse on managing qualitative change in SMEs and hope that it will be well received by the scientific community. In the attempt to deliver a collection of the most current and most interesting studies many individuals have played an important role. Most of all, the quality of this special issue is rooted in the creativity and hard work invested in their contributions by the authors. We are very grateful for their efforts during the multi-stage review process and for sticking to timelines. Special acknowledgement is given to the reviewers for their insightful and constructive comments and the journal editors-in-chief for their support in developing this special issue.

We hope that you will enjoy reading the papers and that this special issue can either answer some questions for you or provide you with some new questions that propel your own future research.

\section{References}

Adler PS, Benner M, Brunner DJ, MacDuffie JP, Osono E, Staats BR, Takeuchi H, Tushman ML, Winter SG (2009) Perspectives on the productivity dilemma. J Oper Manag 27(2):99-113

Cohen MD (2007) Administrative behavior: laying the foundation for Cyert and March. Organ Sci 18(3):503-506

Davidsson P, Achtenhagen L, Naldi L (2010) Small firm growth. Found Trends Entrep 6(2):69-165

March JG (1991) Exploration and exploitation in organizational learning. Organ Sci 2(1):71-87

March JG, Simon H (1958) Organizations. Wiley, New York

Mejia-Morelos J, Grima F, Trepo G (2013) Change and stability interaction processes in SMEs: a comparative case study. J Organ Change Manag 26(2):370-422

Moshe F (2010) Beyond dualism: stability and change as a duality. Acad Manag Rev 35(2):202-225

Nelson RR, Winter S (1982) An evolutionary theory of economic change. Harvard University Press, Cambridge 
Sutherland F, Smith A (2011) Duality theory and the management of the change-stability paradox. J Manag Organ 17(4):534-547

Van de Ven AH, Poole MS (1995) Explaining development and change in organizations. Acad Manag Rev 20(3):510-540

Van Gestel N, Hillebrand B (2011) Explaining stability and change: the rise and fall of logics in pluralistic fields. Organ Stud 32(2):231-252

Weick KE (1979) The social psychology of organizing, 2nd edn. Addison-Wesley, Reading 